\title{
Rhizoeconomics: The Roots of Shoot Growth Limitations
}

\author{
Jonathan P. Lynch \\ Department of Horticulture, The Pennsylvania State University, 102 Tyson Building, University Park, PA 16802
}

\begin{abstract}
The growth of terrestrial plants is primarily limited by edaphic factors such as drought, nutrient deficiencies, and mineral toxicities. Roots express an array of adaptive traits that help plants cope with these stresses, but such traits often incur significant costs, including direct metabolic costs, tradeoffs for contrasting resources, opportunity costs, and increased risks of biotic and abiotic stress. As an example, root cortical aerenchyma appears to improve crop growth under drought and low soil fertility by reducing the metabolic costs of soil exploration. Production of root cortical aerenchyma, however, may involve tradeoffs by reducing radial transport, mycorrhizal colonization, and by increasing disease susceptibility. A better understanding is needed of the full costs associated with specific root traits if we are to develop crops with better growth and yield in the stressful soil environments that increasingly dominate the earth's surface.
\end{abstract}

\section{MOST PLANTS ON EARTH ARE LIMITED BY SOIL CONSTRAINTS}

Edaphic (soil-related) factors are primary limitations to the growth of terrestrial plants. Periodic or seasonal drought is virtually ubiquitous. Few soils provide plant nutrients in optimal amounts, and the most fertile soils are characterized by intense belowground competition. Soils that support the greatest plant biomass are typically highly weathered, acidic, and have potentially toxic amounts of bioavailable aluminum and manganese. Although fertilization has alleviated these constraints in high-input agriculture in rich countries, fertilization is minimal and declining in many food-insecure regions, and globally, soil fertility is declining because of anthropogenic erosion, acidification, and salinization. In general, we do not understand how edaphic stresses will interact with global change, but there is little reason to believe that the situation will improve in the near future, with the possible exception of increasing $\mathrm{N}$ availability in industrial regions caused by acid rain (Lynch and St.Clair, 2004). With the human population expected to reach nine billion by the middle of this century, the development of crops with enhanced tolerance of edaphic stress is an important challenge facing plant biology (Lynch, 2007).

\section{EDAPHIC STRESS TOLERANCE CAN BE EXPENSIVE}

Plants express an array of root traits (a trait being a phenotypic character of an organism) that improve tolerance of edaphic stress, including enhanced soil exploration, enhanced soil resource exploitation, and exclusion or detoxification of toxic minerals. The length of this overview does not permit consideration of the numerous specific adaptations to edaphic stresses or their costs (for a review of costs associated with phosphorus acquisition, see Lynch and Ho, 2005). In general, these costs include:

Direct metabolic costs. These are the resource costs associated with production or maintenance of a trait. An obvious example is the metabolic cost of building and maintaining extensive root systems (including associated microbial symbionts) required for soil foraging for scarce resources. A number of studies have shown that the metabolic costs of soil exploration by root systems are quite substantial and can exceed $50 \%$ of daily photosynthate production (Lambers et al., 2002).

Tradeoffs. Plant traits that may be adaptive in the context of one constraint may be detrimental in the context of another constraint. An example of this is that root architectural traits that enhance topsoil foraging enhance phosphorus acquisition, because phosphorus availability is greatest in the topsoil, at the cost of increased drought sensitivity, because under drought conditions, water availability is typically greatest in the subsoil (Ho et al., 2004, 2005; Lynch and Brown, 2001).

Opportunity costs. These are costs associated with opportunities foregone because of the expression of a trait. An example of this is the allocation of internal resources to root growth and maintenance, which diverts resources from shoot growth and reproduction. In perennial plants, reproduction may be foregone entirely in a stressful season.

Increased risk. Expression of a trait may generate risks of abiotic or biotic stress. For example, delayed phenology (i.e., delayed flowering or maturity) may enhance plant growth under phosphorus stress by increasing time available for soil exploration, soil exploitation, and phosphorus use in the plant (Lynch and Brown, 2006), but delayed phenology may create risks of reproductive failure attributable to late-season frost.

Full consideration of the costs and benefits associated with specific traits is needed if we are to understand plant adaptation to edaphic stress. This is especially important in the selection and breeding of crops for stressful environments. As an example of the importance of root costs in plant adaptation to infertile soil, I consider the case of root cortical aerenchyma.

\section{ROOT CORTICAL AERENCHYMA INCREASES TOLERANCE TO DROUGHT AND LOW NUTRIENT STRESS BY REDUCING THE METABOLIC COSTS OF SOIL EXPLORATION}

Aerenchyma is tissue with large intercellular spaces (Esau, 1977). Root cortical aerenchyma (RCA) replaces living cortical cells with air-filled lacunae (Fig. 1). Although the vast majority of research on RCA has focused on its importance in hypoxia (reviewed in Jackson and Armstrong, 1999), RCA can also be induced by suboptimal availability of nitrogen, phosphorus, and sulfur (Bouranis et al., 2003; Drew et al., 1989; Fan et al., 2003; Konings and Verschuren, 1980). RCA is an important adaptation to soil flooding by facilitating oxygen movement to the roots. We hypothesized that RCA is also an adaptation to low nutrient availability by reducing the metabolic cost of soil exploration (Fan et al., 2003; Lynch and Brown, 1998). By replacing living cells with air, RCA significantly reduces both the respiratory and nutrient requirements of root tissue, permitting greater root growth and nutrient acquisition for a given metabolic investment.

Our research supports the hypothesis that RCA is a useful adaptation to low nutrient availability. In bean and maize, we observed large genetic variation in RCA (Fan et al., 2003). Differences in RCA were correlated with proportionate reductions in root phosphorus concentration (Fig. 2A) and greater than expected reductions in root respiration (Fig. 2B). Root segments with $20 \%$ crosssectional area as RCA had only half the respiration of roots without RCA (Fig. 2B). The disproportionate effect of RCA on respiration may reflect the fact that the cortical cells lost during the formation of RCA are metabolically active, whereas inactive tissues such as xylem vessels, which are not lost in the formation of RCA, do not respire. Results with isolated root segments were confirmed in intact plants (Fan et al., 2003). These results were supported by a field study in which RCA was closely correlated with the ability of maize genotypes to maintain root growth under phosphorus stress (Fig. 3). 

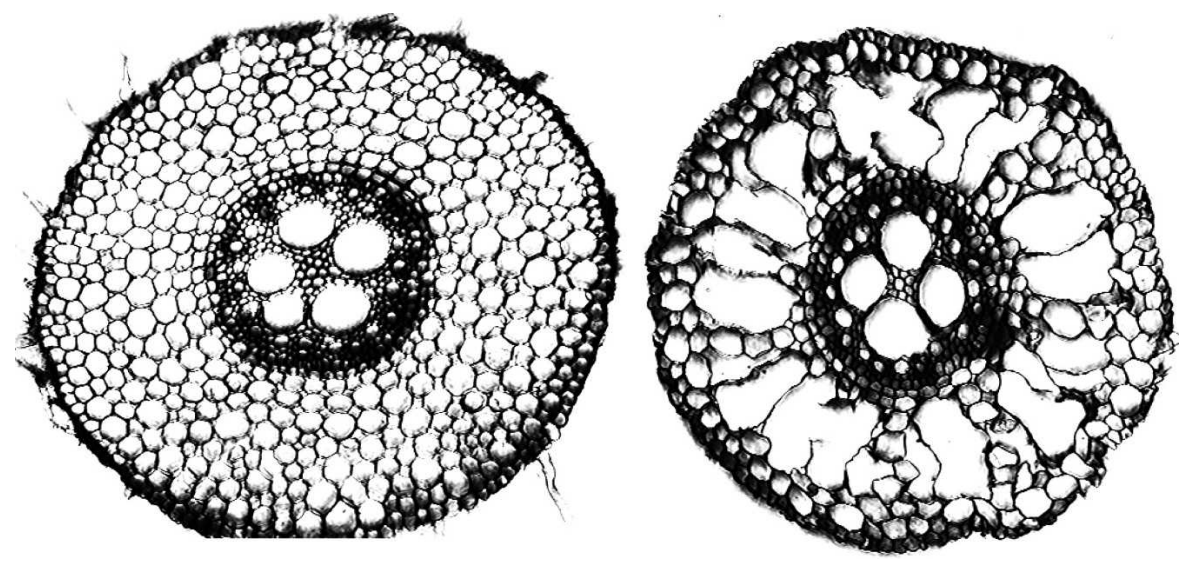

Fig. 1. Cross-sections of seminal roots of maize showing genotypic difference in cortical aerenchyma formation, which replaces living cortical cells (left) with air-tilled lacunae (right). Genotypes are closely related recombinant inbred lines (RILs) of the same parents.

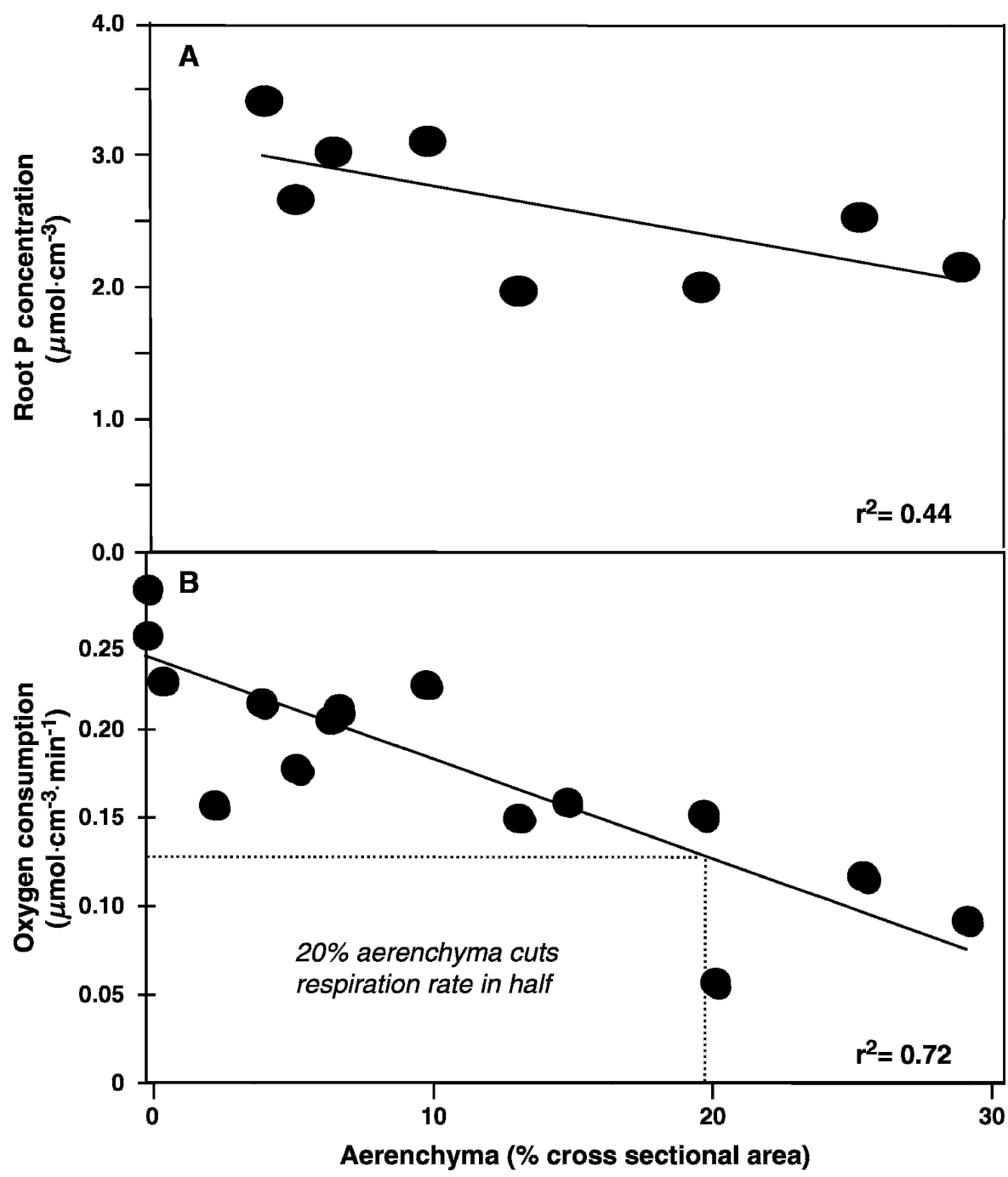

Fig. 2. Reduction of (A) maize root $\mathrm{P}$ concentration and (B) respiration by aerenchyma formation. From Fan et al. (2003).

We have recently obtained evidence that RCA also enhances drought tolerance in maize through a similar mechanism. Six closely related maize genotypes (recombinant inbred lines [RILs] of OH43xW64a) contrasting for RCA (Fig. 1) were planted in large soil cylinders $(1.5 \mathrm{~m} \times 15.24 \mathrm{~cm})$ with and without drought. At planting, the soil columns were at field capacity, and over time, the drought treatment received decreasing irrigation and became progressively drier in the topsoil, as typically occurs in the field.

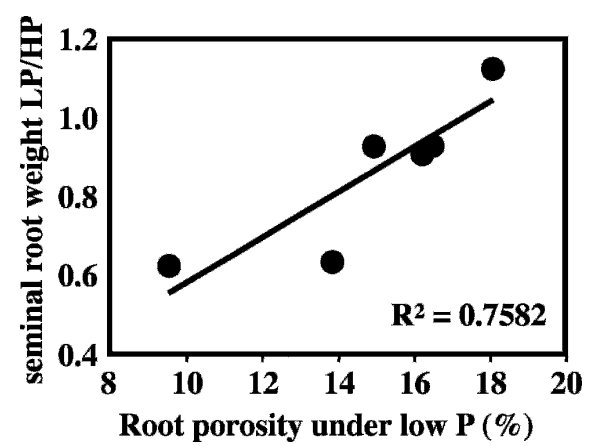

Fig 3. Correlation of aerenchyma with root growth under low $\mathrm{P}$ (as a fraction of root growth in high $\mathrm{P})$ in six maize genotypes in the field.

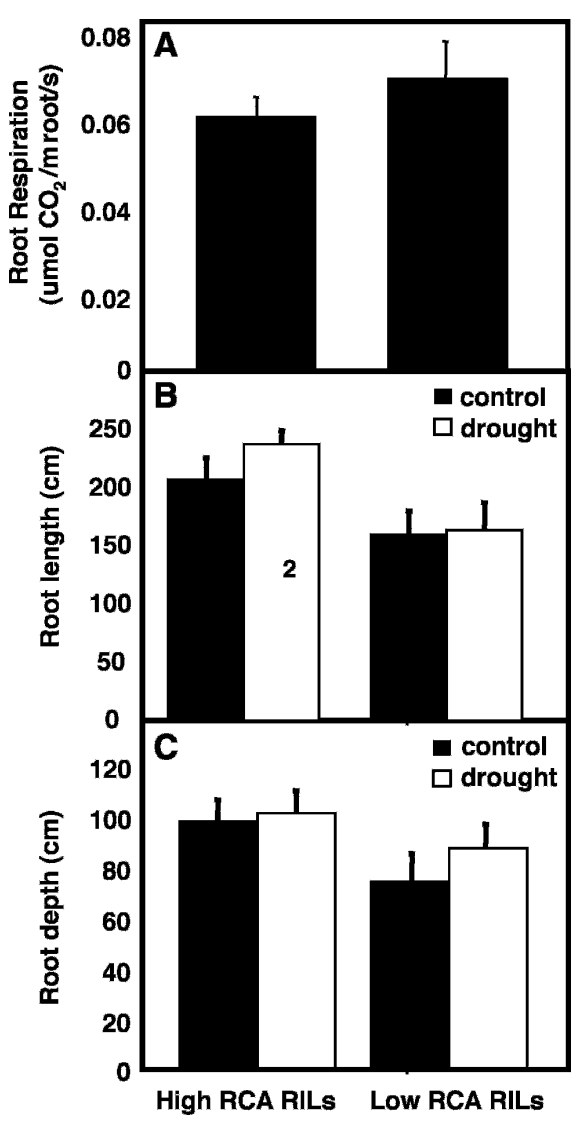

Fig. 4. High aerenchyma (RCA) maize genotypes had less total system respiration per unit root length (A) $\mathrm{F}=4.1, P<0.05$, greater length of the primary root and its laterals; $(\mathbf{B}) \mathrm{F}=10.0$, $P=0.013$, and greater maximum root depth; (C) $\mathrm{F}=112.6, P=<0.001$. Each bar is the mean of 2-4 replicates of 3 RILs harvested 5 weeks after planting, \pm SE.

Genotypes with more RCA had less in situ root respiration (Fig. 4A). By 5 weeks after planting, genotypes with more RCA maintained greater root length (Fig. 4B) and attained greater rooting depth (Fig. 4C) than genotypes with less RCA. Under these conditions, drought stress was just beginning to affect shoot growth (data not shown). We repeated this study with six closely related maize genotypes from the intermated B73xMo17 (IBM) population. Results 

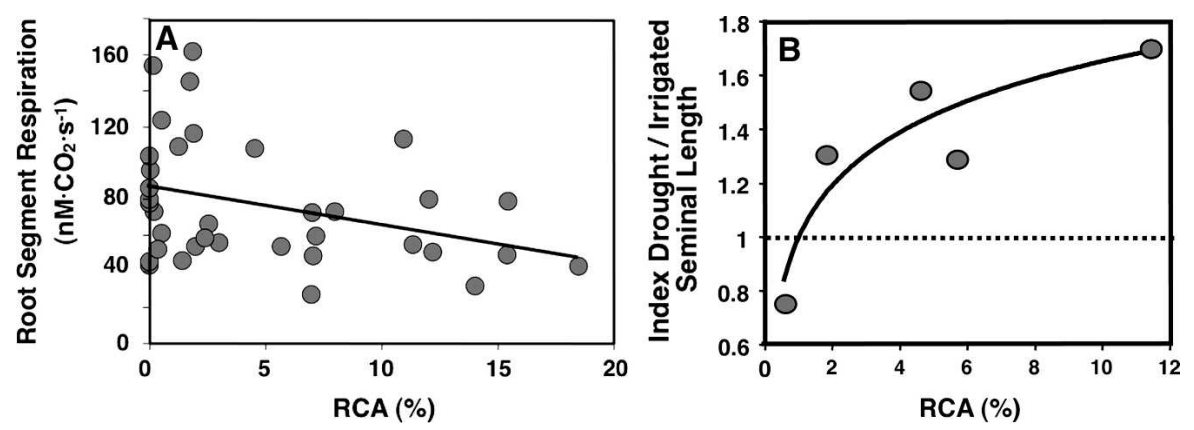

Fig. 5. High root cortical aerenchyma genotypes had lower respiration of nodal roots; $(\mathbf{A}) \mathrm{r}=-0.34, \mathrm{~F}=4.5$. $P=0.04)$ and greater seminal root growth under drought; $(\mathbf{B}) \mathrm{r}=0.92, \mathrm{~F}=15.7, P=0.029)$. Data from six intermated B73xMo17 recombinant inbred lines grown under drought or well-watered conditions for $28 \mathrm{~d}$ in soil mesocosms. In (B), a value of 1.0 means that drought did not affect seminal root length; the value of 1.5 means that drought increased seminal root length by $50 \%$ over well-watered plants.

confirmed previous results and also showed that genotypes with high RCA had lower respiration of nodal root segments (Fig. 5A) and greater seminal root growth under drought stress (Fig. 5B). Screening of 21 IBM RILs showed that increasing RCA was correlated with a $53 \%$ increase in $g_{\mathrm{S}}$ under drought $(\mathrm{F}=3.1, P=0.08)$.

These results indicate that reducing root metabolic costs through RCA formation permits greater root growth and therefore greater acquisition of phosphorus and water under stress conditions. Given the large genotypic variation in RCA in crop germplasm (Fig. 1), selection of high RCA genotypes may be useful in crop-breeding programs. However, before this recommendation can be made to plant breeders, we need to have a better understanding of the costs associated with this trait. The costs of RCA formation are unknown but may include reduced radial transport of nutrients and water, reduced mycorrhizal habitat, and increased susceptibility to the axial spread of root pathogens.

\section{CLOSING THOUGHTS}

The example of root cortical aerenchyma shows the potential use of considering costs in understanding root adaptations to edaphic stress. It also illustrates the surprising knowledge gaps that remain in the areas of whole plant biology and crop ecophysiology, gaps that in general are not easily addressed in the current climate for research funding because they do not require cutting-edge molecular techniques. A better understanding of the total costs and benefits of specific traits for edaphic stress tolerance, and thus their potential value in agriculture, will require a wholistic approach to plant biology. Such an approach encompasses emergent properties of roots, plants, and ecosystems that are not predictable from nucleotide and polypeptide sequences. Given the urgent need for more stress-adapted crops in developing nations, the opportunity costs of not taking this approach are considerable.

\section{Literature Cited}

Bouranis, D.L., S.N. Chorianopoulou, V.F. Siyiannis, V.E. Protonotarios, and M.J. Hawkesford. 2003. Aerenchyma formation in roots of maize during sulphate starvation. Planta 217:382391.

Drew, M., C. He, and P. Morgan. 1989. Decreased ethylene biosynthesis, and induction of aerenchyma, by nitrogen- or phosphate-starvation in adventitious roots of Zea mays L. Plant Physiol. 91:266-271.

Esau, K. 1977. Anatomy of seed plants. John Wiley and Sons, New York.

Fan, M.S., J.M. Zhu, C. Richards, K.M. Brown, and J.P. Lynch. 2003. Physiological roles for aerenchyma in phosphorus-stressed roots. Funct. Plant Biol. 30:493-506.

Ho, M., B. McCannon, and J. Lynch. 2004. Optimization modeling of plant root architecture for water and phosphorus acquisition. J. Theor. Biol. 226:331-340.

Ho, M., J. Rosas, K. Brown, and J. Lynch. 2005. Root architectural tradeoffs for water and phosphorus acquisition. Funct. Plant Biol. 32: 737-748.

Jackson, M.B. and W. Armstrong. 1999. Formation of aerenchyma and the processes of plant ventilation in relation to soil flooding and submergence. Plant Biol. 1:274-287.

Konings, H. and G. Verschuren. 1980. Formation of aerenchyma in roots of Zea mays in aerated solutions, and its relation to nutrient supply. Physiol. Plant. 49:265-279.

Lambers, H., O. Atkin, and F.F. Millenaar. 2002. Respiratory patterns in roots in relation to their functioning, p. 521-552. In: Waisel, Y., A. Eshel, and K. Kafkaki (eds.). Plant roots, the hidden half. Marcel Dekker, Inc., New York.

Lynch, J. and K. Brown. 1998. Root architecture and phosphorus acquisition efficiency in common bean. In: Lynch, J. and J. Deikman (eds.). Phosphorus in plant biology: Regulatory roles in ecosystem, organismic, cellular, and molecular processes. ASPP, Rockville, MD.

Lynch, J. and K. Brown. 2006. Whole plant adaptations to low phosphorus availability. In: Huang, B. (ed.). Plant-environment interactions. 3rd ed. Taylor and Francis, New York.

Lynch, J. and M. Ho. 2005. Rhizoeconomics: Carbon costs of phosphorus acquisition. Plant Soil 269:45-56.

Lynch, J. and S. St.Clair. 2004. Mineral stress: The missing link in understanding how global climate change will affect plants in real world soils. Field Crops Res. 90:101-115.

Lynch, J.P. 2007. Roots of the second green revolution. Aust. J. Bot. (in press).

Lynch, J.P. and K.M. Brown. 2001. Topsoil foraging - An architectural adaptation of plants to low phosphorus availability. Plant Soil 237:225-237. 